DOI: $10.17516 / 1998-2836-0172$

УДК 541.127:547.414

\title{
Thermal Decomposition Kinetics \\ of Bis-Gem-Dinitroethyl Derivatives \\ of N-Nitraminoacetic Acid
}

\author{
Lyudmila A. Kruglyakova*, Rudolf S. Stepanov, \\ Konstantin V. Pekhotin and Oksana A. Golubtsova \\ Reshetnev Siberian State University \\ of Science and Technology \\ Krasnoyarsk, Russian Federation
}

Received 14.12.2019, received in revised form 10.01.2020, accepted 23.02.2020

\begin{abstract}
Thermal decomposition kinetics of bis-gem-dinitroethyl substituted compounds based on N-nitraminoacetic acid is studied. Structural and kinetic patterns are revealed and a chemical mechanism of decomposition reaction is established, including the primary homolysis of $\mathrm{C}-\mathrm{NO}_{2}$ bond in the gem-dinitroethyl fragment. The activation parameters are defined for the initial reaction. The reactivity of the studied compounds was analyzed and quantitative correlations between the logarithms of the rate constant, the activation energies and the steric constants of substituents in the gem-dinitroethyl group were found.
\end{abstract}

Keywords: kinetics, thermal decomposition, gem-dinitroethyl derivatives, mechanism, activation parameters, steric constants of substituent.

Citation: Kruglyakova L.A., Stepanov R.S., Pekhotin K.V., Golubtsova O.A. Thermal decomposition kinetics of bis-gemdinitroethyl derivatives of N-nitraminoacetic acid, J. Sib. Fed. Univ. Chem., 2020, 13(2), 167-174. DOI: 10.17516/1998-28360172

(C) Siberian Federal University. All rights reserved

This work is licensed under a Creative Commons Attribution-NonCommercial 4.0 International License (CC BY-NC 4.0).

* Corresponding author E-mail address: lakruglyakova@sibsau.ru 


\title{
Кинетика термического разложения \\ бис-гем-динитроэтилпроизводных \\ N-нитраминоуксусной кислоты
}

\author{
Л.А. Круглякова, Р.С. Степанов, \\ К.В. Пехотин, О.А. Голубцова \\ Сибирский государственный университет науки и технологий \\ имени академика М.Ф. Решетнева \\ Российская Федерация, Красноярск
}

Аннотация. Изучена кинетика термораспада бис-гем-динитроэтилзамещенных соединений на базе N-нитраминоуксусной кислоты. Раскрыты структурно-кинетические закономерности и установлен химический механизм реакции разложения, включающий первичный гомолиз связи $\mathrm{C}-\mathrm{NO}_{2}$ в гем-динитроэтильном фрагменте. Для первичной реакции определены активационные параметры. Проведен анализ реакционной способности изученных соединений и найдены количественные зависимости между логарифмами константы скорости, энергиями активации и стерическими константами заместителя в гем-динитроэтильной группе.

Ключевые слова: кинетика, термораспад, гем-динитроэтилпроизводные, механизм, активационные параметры, стерические константы заместителя.

Цитирование: Круглякова, Л.А. Кинетика термического разложения бис-гем-динитроэтилпроизводных N-нитраминоуксусной кислоты / Л.А. Круглякова, Р.С. Степанов, К.В. Пехотин, О.А. Голубцова // Журн. Сиб. федер. ун-та. Химия, 2020. 13(2). С. 167-174. DOI: 10.17516/1998-2836-0172

\section{Введение}

Реакционная способность полифункциональных нитросоединений в реакции термического разложения в течение нескольких десятилетий является предметом исследования и представляет практический интерес [1-7]. Для многих моно- и полинитросоединений на базе алифатических, алициклических и ароматических структур установлены кинетические закономерности и лимитирующая стадия реакции. В то же время наличие в молекуле нитросоединения других функциональных групп влияет на скорость термораспада и может приводить к смене реакционного центра. В продолжение работ по влиянию строения на скорость и механизм термораспада вторичных нитраминов [8-10], содержащих гем-динитроэтильные группы, в настоящей работе изучена кинетика термического разложения соединений общей формулы.<smiles>[R]O[R]</smiles>

где $\mathrm{R}=\mathrm{CH}_{3}$ (I); $\mathrm{CH}_{3} \mathrm{~N}\left(\mathrm{NO}_{2}\right) \mathrm{CH}_{2}$ (II); $\mathrm{NO}_{2}$ (III); $\mathrm{Cl}$ (IV);<smiles>O=[N+]([O-])c1ncn(-c2ccccc2)n1</smiles> 


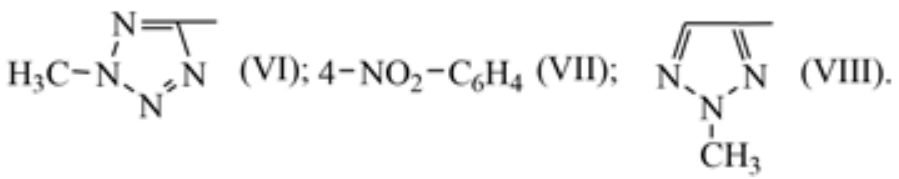

\section{Экспериментальная часть}

Взятые для исследования соединения (I)-(VIII) синтезированы и очищены известными методами [11], были хроматографически чистыми и содержали 99,7-99,8 \% основного вещества.

Кинетику термораспада изучали в изотермических условиях манометрическим методом с использованием стеклянного манометра типа Бурдона при остаточном давлении воздуха в реакционном пространстве манометра $10^{-2}-10^{-1}$ мм рт. ст.

Анализ газообразных продуктов распада проводили на масс-спектрометре MX-1303 при следующих условиях: ионизирующее напряжение 60 эВ, ток эмиссии 2 мА, температура источника ионов $150{ }^{\circ} \mathrm{C}$, температура баллона напуска $110{ }^{\circ} \mathrm{C}$. Конденсированные продукты термораспада идентифицировали на хроматомасс-спектрометре MX-1312 (колонка со скваланом, температура эффузионной камеры $110^{\circ} \mathrm{C}$ ).

Константы скорости термораспада рассчитывали по уравнению первого порядка методом Гуггенгейма. Ошибка в определении константы скорости не превышала 7,5 \%, а энергии активации - 3,9 кДж/моль.

\section{Результаты и их обсуждение}

Для исследования кинетики термораспада использовали низкоплавкий инертный и термостойкий растворитель - дифениловый эфир (ДФЭ), имеющий малую диэлектрическую проницаемость $(\varepsilon=3,7)$. Предварительными опытами было установлено, что концентрация вещества в растворе в пределах 1,0-5,0 \% (масс.) не влияет на скорость термораспада. Поэтому изучение влияния температуры на скорость реакции проводили в 2,0-2,5 \%-ных растворах, где исключены взаимодействия промежуточных и конечных продуктов распада с исходным веществом. В этих условиях реакция распада до степени превращения 40-45 \% подчинялась уравнению первого порядка.

Активационные параметры термораспада, стерические константы заместителей [12] и энтропия активации изученных соединений приведены в таблице.

Из данных таблицы следует, что с увеличением абсолютной величины стерической константы $E_{\mathrm{s}}$ заместителя $\mathrm{R}$ в $\alpha$-положении к геминальной динитроэтильной группе при переходе от соединения (I) к соединению (VIII) происходит возрастание константы скорости термораспада от $0,19 \cdot 10^{-5}$ до $925 \cdot 10^{-5} \mathrm{c}^{-1}$, т.е. в 4868 раз. Это свидетельствует о том, что реакционный центр, на котором инициируется реакция термораспада, расположен на атоме углерода геминальной динитроэтильной группы. Одновременно с увеличением константы скорости происходит уменьшение энергии активации с 173,5 до 139,5 кДж/моль при практически постоянной величине логарифма предэкспоненциального множителя в пределах 15,70-15,19, что хорошо согласуется также со слабым разбросом величины энтропии активации при незначительной сольватации переходного состояния реакции термораспада соединений (I)-(VIII). 
Таблица. Активационные параметры термораспада бис-гем-динитроэтилпроизводных на базе

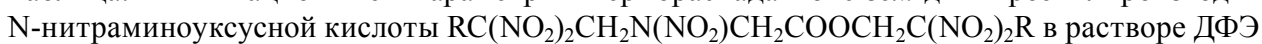

Table. Activation parameters of thermal decomposition of bis-gem-dinitroethyl derivativeson the basis of $\mathrm{N}$-nitraminoaceticacid $\mathrm{RC}\left(\mathrm{NO}_{2}\right)_{2} \mathrm{CH}_{2} \mathrm{~N}\left(\mathrm{NO}_{2}\right) \mathrm{CH}_{2} \mathrm{COOCH}_{2} \mathrm{C}\left(\mathrm{NO}_{2}\right)_{2} \mathrm{R}$ in $\mathrm{DPhE}$ solution

\begin{tabular}{|c|c|c|c|c|c|c|c|}
\hline Шифр & Заместитель & $D T,{ }^{\circ} \mathrm{C}$ & $\begin{array}{c}E_{\mathrm{a}}, \\
\text { кДж/моль }\end{array}$ & $\lg A$ & $\begin{array}{c}k_{150^{\circ}{ }^{\circ} \cdot 10^{5}}, \\
\mathrm{c}^{-1}\end{array}$ & $\begin{array}{c}D S^{\ddagger}{ }_{150^{\circ} \mathrm{C}} \\
\text { Дж/(мольК) }\end{array}$ & $E_{\mathrm{s}}$ \\
\hline I & $\mathrm{CH}_{3}$ & $145-170$ & 173,5 & 15,70 & 0,19 & 44,3 & 0 \\
\hline II & $\mathrm{CH}_{3} \mathrm{~N}\left(\mathrm{NO}_{2}\right) \mathrm{CH}_{2}$ & $130-160$ & 169,2 & 15,63 & 0,55 & 43,9 & $-0,58$ \\
\hline III & $\mathrm{NO}_{2}$ & $120-160$ & 160,3 & 15,55 & 5,73 & 41,5 & $-2,14$ \\
\hline IV & $\mathrm{Cl}$ & $120-160$ & 155,0 & 15,45 & 20,53 & 39,6 & $-2,81$ \\
\hline $\mathrm{V}$ & $3-\mathrm{NO}_{2}-1,2,4-\operatorname{Tr}-1^{*}$ & $120-160$ & 150,2 & 15,39 & 70,00 & 38,4 & $-3,50$ \\
\hline VI & $2-\mathrm{CH}_{3}-\mathrm{Tetr}-5^{* *}$ & $120-150$ & 145,0 & 15,25 & 222,0 & 35,7 & $-4,25$ \\
\hline VII & $4-\mathrm{NO}_{2}-\mathrm{C}_{6} \mathrm{H}_{4}$ & $100-125$ & 141,5 & 15,27 & 630,0 & 36,1 & $-5,10$ \\
\hline VIII & $2-\mathrm{CH}_{3}-1,2,3-\mathrm{Tr}-5^{* * *}$ & $90-110$ & 139,5 & 15,19 & 925,0 & 34,6 & $-5,26$ \\
\hline
\end{tabular}

Примечание: * - 3- $\mathrm{NO}_{2}-1,2,4-\mathrm{Tr}-1$ - 3-нитро-1,2,4-триазол-1-ил;

** - 2- $\mathrm{CH}_{3}$-Tetr-5 - 2-метил-1,2,3,4-тетразол-5-ил;

*** - 2- $\mathrm{CH}_{3}-1,2,3-\mathrm{Tr}-5$ - 2-метил-1,2,3-триазол-5-ил

Для идентификации газообразных и конденсированных продуктов, а также выявления их возможного влияния на скорость и закон разложения на примере соединения (IV) была изучена кинетика реакции термораспада в расплаве при температуре сравнения $\left(150{ }^{\circ} \mathrm{C}\right)$. Оказалось, что до степени превращения 48 \% термораспад, как и в растворе, можно описать уравнением первого порядка. Скорость термораспада не зависит от величины $\mathrm{m} / \mathrm{V}$ (отношения массы вещества к объему реакционного сосуда) и $\mathrm{S} / \mathrm{V}$ (отношения поверхности реакционного сосуда к его объему). Все это свидетельствует об отсутствии на стенках реакционного сосуда гетерогенных и цепных процессов, а также указывает на гомогенность реакции распада. Было показано, что добавки газообразных продуктов, образующихся при распаде в расплаве при глубине превращения 18-22 \%, к свежему веществу не влияют на скорость термораспада.

В газообразных продуктах термораспада соединения (IV) были обнаружены в основном $\mathrm{NO}, \mathrm{N}_{2} \mathrm{O}, \mathrm{N}_{2}, \mathrm{NH}_{3}, \mathrm{CO}, \mathrm{CO}_{2}, \mathrm{H}_{2} \mathrm{O}, \mathrm{HCN}$ (следы), а в конденсированном остатке $\mathrm{NCCH}_{2} \mathrm{~N}_{\left(\mathrm{NO}_{2}\right)}$ $\mathrm{CH}_{2} \mathrm{COOCH}_{2} \mathrm{C}\left(\mathrm{NO}_{2}\right)_{2} \mathrm{Cl}$ (следы), $\mathrm{NCCH}_{2} \mathrm{~N}\left(\mathrm{NO}_{2}\right) \mathrm{CH}_{2} \mathrm{COOH},\left(\mathrm{HOOCCH}_{2}\right)_{2} \mathrm{~N}\left(\mathrm{NO}_{2}\right), \mathrm{NCCH}_{2} \mathrm{~N}=\mathrm{CH}_{2}$, $\mathrm{HOOCCH}_{2} \mathrm{~N}=\mathrm{CH}_{2}$.

Если принять во внимание относительно большое изменение энергии активации при переходе от соединения (I) к соединению (VIII) и небольшую положительную энтропию активации переходного состояния, то можно по аналогии с динитроэтиловыми эфирами $\mathrm{N}$-нитраминоуксусной кислоты на базе 3-нитро-1,2,4-триазола [8] заключить, что термораспад протекает гомолитически и основной вклад в константу скорости вносит энтальпийная составляющая. Реакция инициируется на атоме углерода гем-динитроэтильной группы, расположенной со стороны нитраминного фрагмента. В рамках гомолиза хорошо объясняется образование найденных на опыте продуктов термораспада соединения (IV), где лимитирующей стадией в химическом механизме является разрыв связи С- $\mathrm{NO}_{2}$ : 


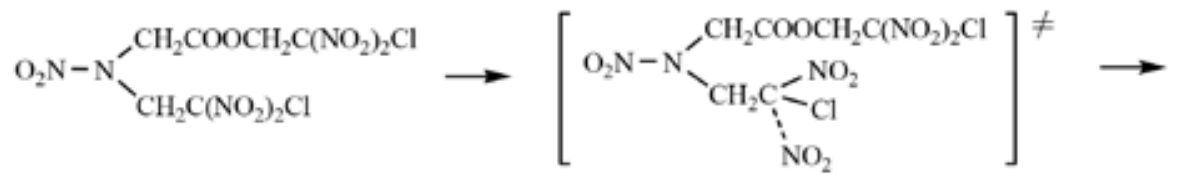

$$
\begin{aligned}
& \rightarrow \mathrm{O}_{2} \mathrm{~N}-\mathrm{N}^{-} \underset{\mathrm{CH}_{2} \dot{\mathrm{C}}\left(\mathrm{NO}_{2}\right) \mathrm{Cl}}{\left.-\mathrm{CH}_{2} \mathrm{COOCH}_{2} \mathrm{CNO}_{2}\right)_{2} \mathrm{Cl}}+\mathrm{NO}_{2}
\end{aligned}
$$

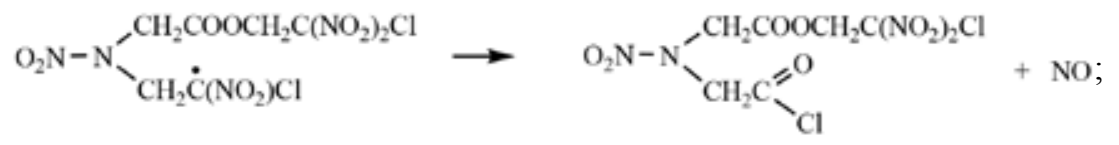

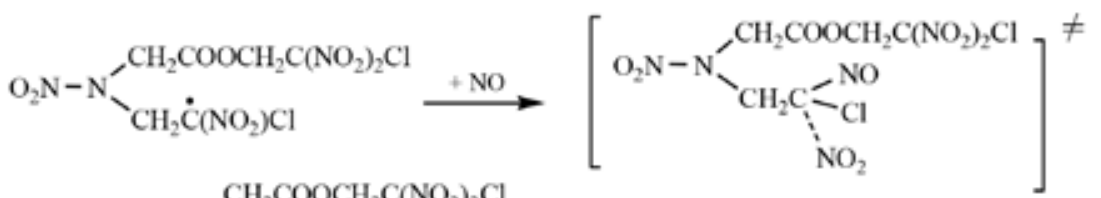

$$
\begin{aligned}
& \longrightarrow \mathrm{O}_{2} \mathrm{~N}-\mathrm{N}^{-} \overbrace{\mathrm{CH}_{2} \dot{\mathrm{C}}_{(\mathrm{NO}) \mathrm{Cl}}}^{-\mathrm{CH}_{2} \mathrm{COOCH}_{2} \mathrm{C}_{\left(\mathrm{NO}_{2}\right)_{2} \mathrm{Cl}}}+\mathrm{NO}_{2} \text {; }
\end{aligned}
$$

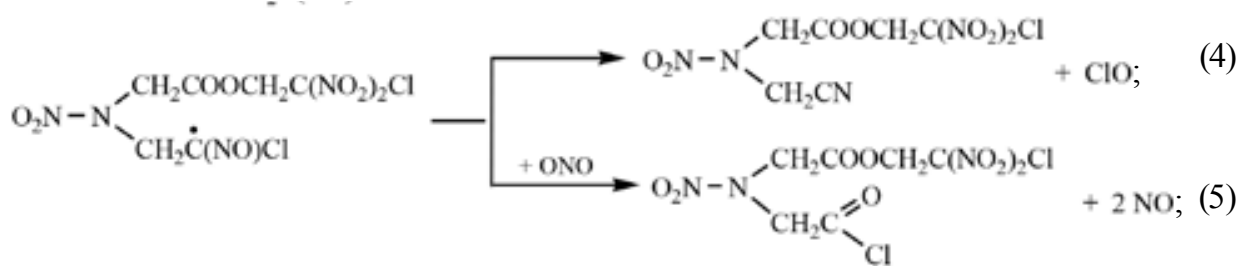

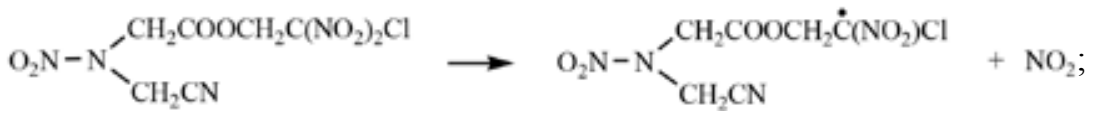

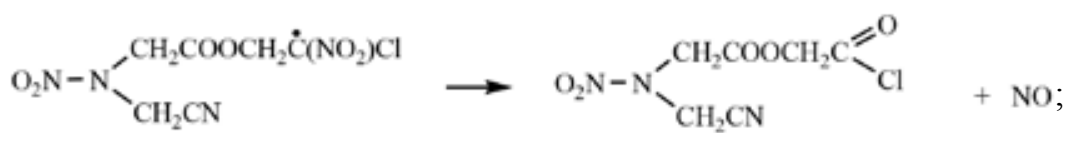

$$
\begin{aligned}
& \mathrm{O}_{2} \mathrm{~N}-\mathrm{N}^{-}{ }_{\mathrm{CH}_{2} \mathrm{CN}}^{-\mathrm{CH}_{2} \mathrm{COOCH}_{2} \mathrm{C}}={ }_{\mathrm{Cl}}^{\mathrm{O}}+\mathrm{NO}_{2} \longrightarrow \mathrm{O}_{2} \mathrm{~N}-\mathrm{N}^{-}-\mathrm{CH}_{2}{ }_{\mathrm{CH}_{2} \mathrm{CN}}^{-\mathrm{COO}^{\circ}}+\mathrm{CH}_{2} \mathrm{O}+\dot{\mathrm{COCl}} \\
& 2 \dot{\mathrm{COCl}} \longrightarrow 2 \mathrm{CO}+\mathrm{Cl}_{2} \text {; } \\
& \mathrm{O}_{2} \mathrm{~N}-\mathrm{N}_{-\mathrm{CH}_{2} \mathrm{CN}}^{-\mathrm{CH}_{2} \mathrm{COO}} \longrightarrow \mathrm{CO}_{2}+\mathrm{NO}_{2}+\mathrm{CH}_{2}=\mathrm{N}-\mathrm{CH}_{2} \mathrm{CN} \text {; } \\
& \mathrm{O}_{2} \mathrm{~N}-\mathrm{N}^{-} \succ_{\mathrm{CH}_{2} \mathrm{CN}}^{-\mathrm{CH}_{2} \mathrm{COO}}+\mathrm{R}_{1} \mathrm{H} \longrightarrow \mathrm{O}_{2} \mathrm{~N}-\mathrm{N}^{-} \succ_{\mathrm{CH}_{2} \mathrm{CN}}^{-\mathrm{CH}_{2} \mathrm{COOH}}+\dot{\mathrm{R}}_{1} \\
& \mathrm{CH}_{2} \mathrm{O}+\mathrm{NO}_{2} \longrightarrow \mathrm{H}_{2} \mathrm{O}+\mathrm{CO}_{2}+\mathrm{NO} \text {; } \\
& \mathrm{O}_{2} \mathrm{~N}-\mathrm{N}^{-}{ }_{\mathrm{CH}_{2} \mathrm{CN}}^{-\mathrm{CH}_{2} \mathrm{COOH}}+2 \mathrm{H}_{2} \mathrm{O} \longrightarrow \mathrm{O}_{2} \mathrm{~N}-\mathrm{N}^{-}{ }_{\mathrm{CH}_{2} \mathrm{COOH}}^{-\mathrm{CH}_{2} \mathrm{COOH}} ;+\mathrm{NH}_{3} \text {; } \\
& \mathrm{CH}_{2}=\mathrm{N}-\mathrm{CH}_{2} \mathrm{CN}+2 \mathrm{H}_{2} \mathrm{O} \longrightarrow \mathrm{CH}_{2}=\mathrm{N}-\mathrm{CH}_{2} \mathrm{COOH}+\mathrm{NH}_{3} \text {; } \\
& \dot{\mathrm{R}}_{1}+\dot{\mathrm{R}}_{1} \quad \longrightarrow \quad \mathrm{R}_{1}-\mathrm{R}_{1} \text {. }
\end{aligned}
$$


Для количественного описания реакционной способности соединений (I)-VIII) в зависимости от природы заместителя R построен график (рис. 1), на котором видно, что логарифмы констант скорости распада хорошо коррелируют со стерическими константами заместителей $E_{\mathrm{s}}$, уравнение регрессии имеет вид

$$
\begin{aligned}
& \lg k_{150^{\circ} \mathrm{C}}=-(0,699 \pm 0,011) E_{\mathrm{s}}-(5,173 \pm 0,034), \\
& \mathrm{r}=0,998 ; \mathrm{S}_{\mathrm{y}}=0,153 ; \mathrm{n}=8 .
\end{aligned}
$$

Аналогичная картина наблюдается для энергии активации (рис. 2).

$$
\begin{aligned}
& E_{\mathrm{a}}=(6,42 \pm 0.13) E_{\mathrm{s}}+(173,24 \pm 0,45), \\
& \mathrm{r}=0,997 ; \mathrm{S}_{\mathrm{y}}=1,76 ; \mathrm{n}=8 .
\end{aligned}
$$

Уравнения (16)-(17) дают возможность оценивать термическую стабильность неизученных гем-динитроэтильных производных на базе $\mathrm{N}$-нитраминокислот, а также представляют

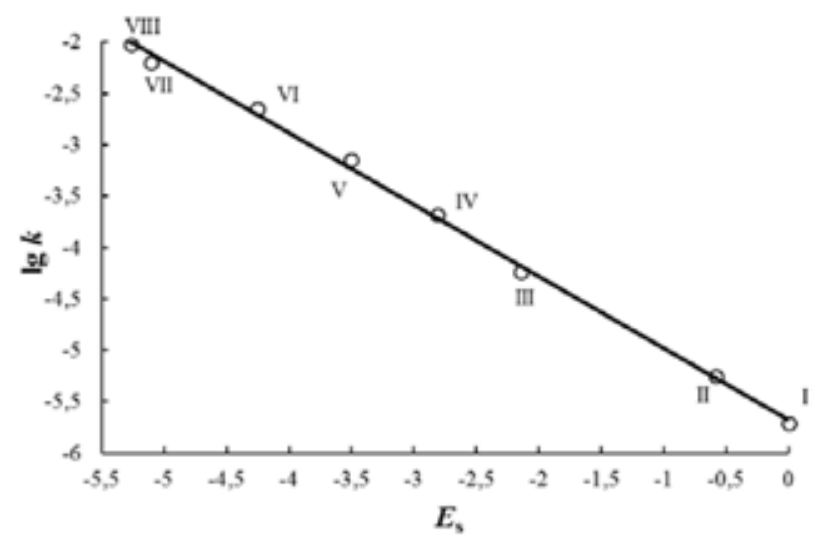

Рис. 1. Зависимость $\lg k_{150}{ }^{\circ} \mathrm{C}=\mathrm{f}\left(E_{\mathrm{s}}\right)$ для соединений (I)-(VIII). Номера точек соответствуют номерам соединений в таблице

Fig. 1. Dependence $\lg k_{150^{\circ} \mathrm{C}}=\mathrm{f}\left(E_{\mathrm{s}}\right)$ for compounds (I)-(VIII). The numbers of points corresponds to compound's number in the table

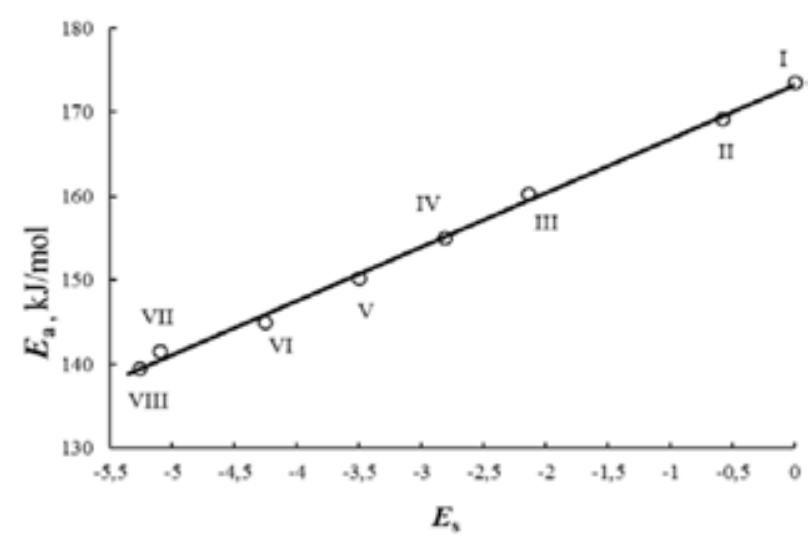

Рис. 2. Зависимость $E_{\mathrm{a}}=\mathrm{f}\left(E_{\mathrm{s}}\right)$ для соединений (I)-(VIII)

Fig. 2. Dependence $E_{\mathrm{a}}=\mathrm{f}\left(\mathrm{S} E_{\mathrm{s}}\right)$ for compounds (I)-(VIII) 
интерес для целенаправленного синтеза соединений этого ряда с заданными параметрами по термостабильности.

\section{Заключение}

Проведенное исследование показало, что термическое разложение бис-гемдинитроэтилпроизводных $\mathrm{N}$-нитраминоуксусной кислоты в растворах инертных растворителей протекает по реакции первого порядка и лимитируется гомолитическим разрывом связи C-NO $\mathrm{NO}_{2}$ в гем-динитрогруппе. На основании анализа газообразных и конденсированных продуктов разложения установлен химический механизм реакции. Природа заместителя в $\alpha$-положении оказывает влияние на реакционную способность соединений. Найдены корреляционные зависимости между стерическими константами заместителей $\mathrm{E}_{\mathrm{s}}$ и константами скорости (энергиями активации) термораспада, обладающие прогнозирующей силой.

\section{Список литературы / References}

1. Смирнов Л.П. Химическая физика разложения энергетических материалов. Проблемы и перспективы. Успехи химии 2004. Т. 71(11), C. 1210-1232. [Smirnov L.P. Chemical Physics of decomposition of energetic materials. Problems and prospects. Russian Chemical Reviews 2004. Vol. 71(11), P. 1121-1141 (in Russ.)].

2. Шу Ю., Корсунский Б.Л., Назин Г.М. Механизм термического разложения вторичных нитраминов. Успехи химии 2004. T. 73(3), C. 320-335. [Shu Yu, Korsounskii B.L., Nazin G.M. The mechanism of thermal decomposition of secondary nitramines. Russian Chemical Reviews 2004. Vol. 73(3), P. 293-307 (in Russ.)].

3. Zeman S. Characteristics of thermal decomposition of energetic materials in a study of their initiation reactivity. Handbook of Thermal Analysis and Calorimetry 2018. Vol. 6, P. 573-612.

4. Pan Y., Zhu W., \& Xiao H. DFT studies on trinitromethyl- or dinitromethyl-modified derivatives of RDX and $\beta$-HMX. Computational and Theoretical Chemistry 2013. Vol. 1019, P. 116124.

5. Kruglyakova L.A., Stepanov R.S. On the mechanism of autocatalytic thermal decomposition of some secondary nitramines. Russian Journal of General Chemistry 2017. Vol. 87(2), P. 204-207.

6. Sinditskii V.P., Hoang T.H., Smirnova A.D., Egorshev V.Y., Yudin N.V., Vatsadze I.A., Dalinger I.L. Comparative study of thermal stability and combustion of dinitropyrazole isomers. Thermochimica Acta 2018. Vol. 667, P. 1-8.

7. Oxley J.C., Smith J.L., Donnelly M.A., Colizza K., \& Rayome S. Thermal Stability Studies Comparing IMX-101 (Dinitroanisole/Nitroguanidine/NTO) to Analogous Formulations Containing Dinitrotoluene. Propellants, Explosives, Pyrotechnics 2015. Vol. 41(1), P. 98-113.

8. Krugliakova L.A., Stepanov R.S. Kinetics and mechanism of thermal decomposition of R-gem-dinitroethyl esters of N-nitraminoacetic acid on the basis of 3-nitro-1,2,4-triazole. Energetic Materials - Particles, Processing, Application. 46 ${ }^{\text {th }}$ International ICT-Conference 2015. Karlsruhe. FRG. P. 66/1-66/6.

9. Kruglyakova L.A., Stepanov R.S., Kekin Yu.V., Pekhotin K.V. Kinetics of Thermal Decomposition of 3,7-Dinitro-1,3,5,7-tetraazabicyclo[3.3.1]nonane. Russian Journal of General Chemistry 2019. Vol. 89(2), P. 194-198. 
10. Krugliakova L.A., Stepanov R.S. Kinetics and mechanism of thermal decomposition of 2,4-dinitro-2,4-diaza-6-R-6,6-dinitrohexane. Proc. $18^{\text {th }}$ International Seminar ${ }^{2} \mathrm{New}$ Trends in Research of Energetic Materials² 2015. Pardubice. The Czech Republic. P. 659-663.

11. Николаев В.Д., Ищенко М.А. Ацетали и сложные эфиры полинитроспиртов. Российский химический журнал 1997. Т. 41(2), C. 14-21. [Nikolaev V.D., Ishchenko M.A. Acetals and esters of polynitroalcohols. Russian Chemical Journal [Mendeleev Chemical Journal] 1997. Vol. 41(2), P. 14 21. (in Russ.)]

12. Kruglyakova L.A., Stepanov R.S., Golubtsova O.A., Pekhotin K.V. Effect of additive polyfunctional substituents on the thermal decomposition rate of $\mathrm{R}^{1}, \mathrm{R}^{2}, \mathrm{R}^{3}$-nitromethanes. Russian Journal of General Chemistry 2015. Vol. 84(8), P. 1500-1504. 